\title{
Assurer la sécurité des contraceptifs par des chaînes d'approvisionnement efficacies
}

Moazzam Ali

Follow this and additional works at: https://knowledgecommons.popcouncil.org/departments_sbsr-rh

Part of the Social and Behavioral Sciences Commons How does access to this work benefit you? Let us know!

\section{Recommended Citation}

Ali, Moazzam. 2017. "Assurer la sécurité des contraceptifs par des chaînes d'approvisionnement efficacies," Résumés des preuves sur la planification familiale. New York: Population Council. 


\section{Assurer la sécurité des contraceptifs par des chaînes d'approvisionnement efficaces}

\section{$\rightarrow$ Le renforcement des chaînes d'approvisionnement pour répondre à la demande croissante de planification} familiale exige le diagnostic des systèmes, la restructuration ou l'ajustement de la chaîne d'approvisionnement, des systèmes de stockage et de distribution stratégiquement situés, une dotation en personnel et une formation adéquate, ainsi que de meilleures informations sur l'inventaire et le financement.

L'établissement et le maintien de chaînes d'approvisionnement performantes qui répondent aux besoins des secteurs public et privé, ainsi qu'aux professionnels de la santé à tous les niveaux des programmes de planification familiale (PF), joueront un rôle essentiel dans la réduction des besoins non satisfaits en matière de contraception moderne dans les pays à faible revenu et à revenu intermédiaire (PFR-PRI) La gestion de la chaîne d'approvisionnement comprend les étapes de transfert d'un produit du fournisseur au client (1). Le manque d'accès et les défaillances de la chaîne d'approvisionnement sont les principales raisons invoquées par les femmes des PFR-PRI pour la non utilisation de produits contraceptifs et l'abandon de la contraception $(2,3,4)$.

Des chaînes d'approvisionnement efficaces améliorent la qualité des soins et aident les femmes à choisir les méthodes modernes de contraception en réduisant les ruptures de stocks de produits et de matériel médicaux (5). Les insuffisances des chaînes d'approvisionnement sont attribuables à différentes causes, dont la faible demande des clientes et le manque de personnel qualifié. Le nombre de pays où au moins $85 \%$ des points de prestation de services primaires ont offert trois méthodes ou plus a augmenté de 7 en 2013 à 20 en 2015 (6).

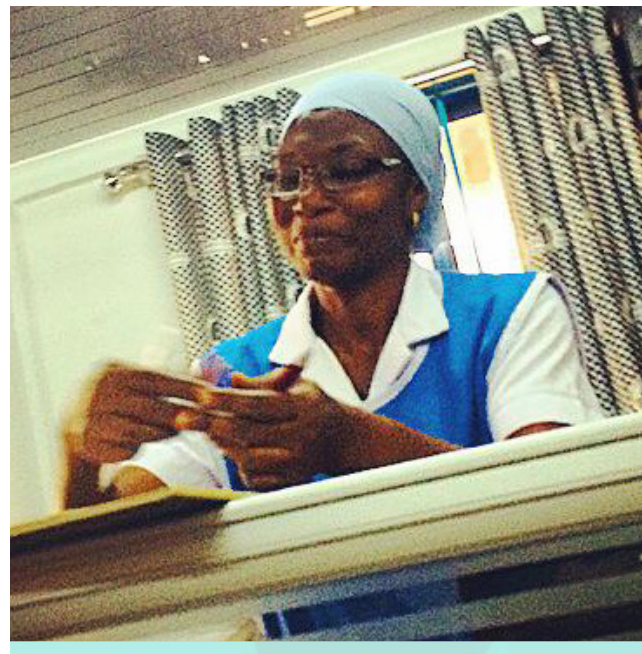

\section{CONSIDÉRATIONS POLITIQUES ET PROGRAMMATIQUES}

Établir et maintenir des chaînes d'approvisionnement efficaces pour les contraceptifs :

$\rightarrow$ Améliorer l'information logistique pour la gestion des stocks; utiliser la technologie mobile pour améliorer le reporting entre les niveaux de la chaîne d'approvisionnement ; et envisager différents modèles de chaîne d'approvisionnement conçus pour traiter les opérations inefficaces.

$\rightarrow$ Tirer parti de l'expertise du secteur privé et du secteur public pour assurer une approche globale du marché de la gestion de la chaîne d'approvisionnement.

$\rightarrow$ Renforcer la capacité des prestataires et des gestionnaires de planification familiale du secteur public et privé et créer des possibilités de collaboration plus étroite. 


\section{OBSTACLES ET DÉFIS}

Plusieurs facteurs contribuent à la faiblesse des chaînes d'approvisionnement dans les PFR-PRI, en particulier dans les régions rurales et périphériques où les clientes sont difficiles à atteindre. Les obstacles sont notamment :

- La faiblesse des systèmes d'information sur la gestion logistique et l'insuffisance des informations sur les stocks, le financement et les flux de produits pour orienter les achats $(7,8,9,10)$.

- L'insuffisance des infrastructures, notamment l'insuffisance des réseaux routiers et l'absence d'installations de stockage appropriées $(8,9,10,11)$.

- L'absence d'une base de ressources humaines stable et bien formée consacrée à la gestion de la chaîne d'approvisionnement $(8,9,11,12,13)$.

- Un financement national et international insuffisants pour l'achat des produits de base $(7,9,10)$.

Le renforcement des chaînes d'approvisionnement pour répondre à la demande croissante de PF nécessitera le diagnostic des systèmes, la restructuration ou l'ajustement de la chaîne d'approvisionnement, des systèmes de stockage et de distribution stratégiquement situés, une dotation en personnel et une formation adéquate, ainsi que de meilleures informations sur l'inventaire et le financement. Une chaîne d'approvisionnement efficace et efficiente aide les femmes à atteindre leurs objectifs en matière de reproduction (14).

Les chaînes d'approvisionnement efficaces peuvent également créer et répondre à la demande latente pour des méthodes jusque-là indisponibles, comme l'illustre l'utilisation par le Sénégal du modèle push éclairé (Informed Push Model - IPM), où des augmentations marquées de 11 points de pourcentage ont été enregistrées en un an dans la région de Dakar en raison de la disponibilité fiable des produits (9).

\section{CONSIDÉRATIONS POUR ASSURER LA SÉCURITÉ DES CONTRACEPTIFS PAR DES CHAÎNES D'APPROVISIONNEMENT EFFICACES}

Deux solutions fondées sur des données probantes répondent aux défis décrits ci-dessus :

- Accroître la visibilité des flux de produits et la demande des utilisateurs. Améliorer l'information logistique pour la gestion des stocks; utiliser la technologie mobile pour améliorer les rapports entre les niveaux de la chaîne d'approvisionnement (15) ; envisager différents modèles de chaîne d'approvisionnement conçus pour traiter les opérations inefficaces; et réévaluer les systèmes « pull » s'ils sont inefficaces (9).

- Tirer parti de l'expertise du secteur privé et du secteur public pour assurer une approche globale du marché de la gestion de la chaîne d'approvisionnement. Renforcer les capacités des fournisseurs et des gestionnaires du secteur privé et du secteur public de la PF et créer des possibilités de collaboration plus étroite $(8,11,12,13,16,17,18)$.

Dans le contexte de l'optimisation de la chaîne d'approvisionnement, les solutions suivantes ont été adoptées de manière novatrice :

- Mise en œuvre de plates-formes nationales de collaboration ou de VAN (Réseaux de visibilité et d'analyse) de personnes, de processus et de technologie qui captent et utilisent les données de la chaîne d'approvisionnement provenant de sources multiples pour améliorer la prise de décision (6).

- Création d'un réseau mondial de visibilité et d'analyse (Global VAN) qui rationalise l'accès aux données nationales et réduit les flux de données parallèles, tout en captant les données des fabricants, des acquéreurs, des transitaires et des autres dans la chaîne d'approvisionnement. Le mécanisme Global VAN facilite l'harmonisation des données et fournit une plateforme unique pour obtenir les données nécessaires pour assurer la fourniture de produits aux programmes de pays (19). L'adoption de normes communes de données et les options de «traçage et de suivi » des produits de santé faciliteront les mesures mondiales $(20,21)$.

- L'adoption du modèle d'incitation à la distribution des contraceptifs au Sénégal et au Togo a permis d'accroître l'efficacité opérationnelle et la possibilité d'augmenter les quantités de fournitures pour stimuler la demande et l'utilisation $(9,22)$.

- L'engagement des gouvernements de l'Éthiopie, du Malawi et du Rwanda pour le renforcement des chaînes d'approvisionnement des produits contraceptifs modernes a démontré que les plus grands avantages sont réalisés lorsque le flux de produits, le flux de données et les personnes efficaces sont en place et travaillent ensemble (8).

- Le renforcement des chaînes d'approvisionnement implique des activités politiques et programmatiques telles que l'intégration et la décentralisation. Le gouvernement chilien utilise des contrats-cadres pour mettre en commun les achats de médicaments essentiels dans un système de santé décentralisé. Ces contrats épargnent au gouvernement chilien près de 8000 USD par achat dans les coûts de gestion et créent une chaîne d'approvisionnement plus souple (23).

- L'intégration des services de planification familiale dans d'autres services de santé est prometteuse et entraîne souvent la fusion de chaînes d'approvisionnement séparées (ou verticales) dans le cadre de vastes réformes du secteur de la santé. Le Ministère de la Santé en Mongolie, grâce à un accord de partenariat stratégique à long terme avec le secteur privé, traite de tous les produits de santé et de la réglementation des frais de service. Cette action stratégique constitue un pas utile vers le renforcement des partenariats publics et privés dans le secteur de la santé et l'amélioration de la chaîne nationale d'approvisionnement en santé (5). 
- Enfin, pour être efficaces et efficientes, les interventions de la chaîne d'approvisionnement doivent obtenir l'engagement politique des gouvernements nationaux ; la participation active de toutes les parties prenantes ; des ressources financières et humaines adéquates ; la qualité et la quantité appropriées des produits; une gestion logistique efficace, y compris les prévisions, le contrôle des stocks, la distribution et les données.

\section{RÉFÉRENCES}

1 Jacoby D. Guide to supply chain management: how getting it right boosts corporate performance. John Wiley \& Sons; 2009.

2 Alkema L, Kantorova V, Menozzi C, Biddlecom A. National, regional, and global rates and trends in contraceptive prevalence and unmet need for family planning between 1990 and 2015: a systematic and comprehensive analysis. Lancet. 2013;381(9878):1642-52.

3 Bradley J, Mursagulova N, Nosa M, Searing H. Supply and demand challenges to modern contraceptive use in Azerbaijan. Eur J Contracept Reprod Health Care. 2007;12(2):175-83.

4 Sedgh G, Hussain R. Reasons for contraceptive nonuse among women having unmet need for contraception in developing countries. Stud Fam Plann. 2014;45(2):151-69.

5 High Impact Practices in Family Planning (HIP). Supply chain management: investing in contraceptive security and strengthening health systems. Washington, DC: USAID; 2012 novembre. Disponible à: http://www.fphighimpactpractices.org/resources/supply-chainmanagement-investing-contraceptivesecurity-and-strengtheninghealth-systems (Accessed on June 20, 2017).

6 UNFPA supplies. Annual report 2015. https://drive.google.com/ file/d/OB9D17AOk5PiRclAtTIZWUnR4SmM/view.

7 Chandani Y, Breton G. Contraceptive security, information flow, and local adaptations: family planning Morocco. Afr Health Sci. 2001;1(2):73-82.

8 Chandani Y, Andersson S, Heaton A, Noel M, Shieshia M, Mwirotsi A, et al. Making products available among community health workers: Evidence for improving community health supply chains from Ethiopia, Malawi, and Rwanda. J Glob Health. 2014;4(2):020405.

9 Daff BM, Seck C, Belkhayat H, Sutton P. Informed push distribution of contraceptives in Senegal reduces stockouts and improves quality of family planning services. Glob Health Sci Pract. 2014;2(2):245-52

10 Vledder M, Friedman J, Sjöblom M, Brown T, Yadav P. Optimal supply chain structure for distributing essential drugs in low income countries: Results from a randomized experiment. Ross School of Business Paper. 2015;1269.

11 Shieshia M, Noel M, Andersson S, Felling B, Alva S, Agarwal S, et al. Strengthening community health supply chain performance through an integrated approach: Using mHealth technology and multilevel teams in Malawi. J Glob Health. 2014;4(2):020406.

12 Prata N, Gessessew A, Cartwright A, Fraser A. Provision of injectable contraceptives in Ethiopia through communitybased reproductive health agents. Bull World Health Organ. 2011;89(8):556-64.

13 Lebetkin E, Orr T, Dzasi K, Keyes E, Shelus V, Mensah S, et al. Injectable contraceptive sales at licensed chemical seller shops in Ghana: access and reported use in rural and periurban communities. Int Perspect Sex Reprod Health. 2014;40(1):21-7.

14 EngenderHealth. 2011. The SEED Assessment Guide for Family Planning Programming. New York.

15 J. Barrington, O. Wereko-Brobby, P. Ward, W. Mwafongo, S. Kungulwe. SMS for Life: a pilot project to improve antimalarial drug supply management in rural Tanzania using standard technology. Malar J, 9 (1) (2010), p. 298 http://dx.doi. org/10.1186/1475-2875-9-298

16 Akol A, Chin-Quee D, Wamala-Mucheri P, Namwebya JH, Mercer SJ, Stanback J. Getting closer to people: family planning provision by drug shops in Uganda. Glob Health Sci Pract. 2014;2(4):472-81.

17 Prata N, Weidert K, Fraser A, Gessessew A. Meeting rural demand: a case for combining community-based distribution and social marketing of injectable contraceptives in Tigray, Ethiopia. PLoS One. 2013;8(7):e68794.
18 Stanback J, Mbonye AK, Bekiita M. Contraceptive injections by community health workers in Uganda: a nonrandomized community trial. Bull World Health Organ. 2007;85(10):768-73.

19 Reproductive Health Supplies Coalition. https://www.rhsupplies. org/fileadmin/uploads/rhsc/Newsletters/Supplylnsider/2016-12Supply-Insider-English.pdf (Accédé le 20 juin, 2017)

20 Reproductive Health Supplies Coalition. https://www.rhsupplies. org/fileadmin/uploads/rhsc/General_Membership_Meetings/ Mexico_2014/Presentations/Day_1/SSWG_-_Panel_1_-_iSCM_ D4M_-_Campbell_Bright_-_UNFPA.pdf (Accédé le 20 juin, 2017)

21 USAID. Deliver Project. Final country report. Ethiopia. 2016. http:// deliver.jsi.com/wp-content/uploads/2017/01/FinaCounRepo_ET.pdf (Accédé le 20 juin, 2017)

22 Reproductive Health Supplies Coalition. https://www.rhsupplies. org/fileadmin/uploads/rhsc/General_Membership_Meetings/ Mexico_2014/Presentations/Day_4/PS_Availability_-_High_ Impact_SCM_Practices_-_Renee_van_der_Weerdt_UNFPA.pdf (Accédé le 20 juin, 2017)

23 Goya F. Balance y Perspectivas de los convenios marco. Présenté au VI Annual Conference on Government Procurement in the Americas; Lima, Pérou, octobre 2010.

Auteur : Moazzam Ali, Organisation mondiale de la Santé. Soutien fourni par Madeline Farron.

Ce document fait partie de l'un des sept Résumés des preuves sur la planification familiale préparés dans le cadre du Sommet de la planification familiale tenu à Londres le 11 juillet 2017. Les résumés mettent en exergue les éléments de preuve et fournissent des considérations sur la recherche et les programmes pour améliorer l'accès à la planification familiale et réduire les grossesses non désirées. Les considérations relatives aux programmes sont basées sur les opinions expertes des auteurs, qui ont procédé à des examens documentaires sur la base des preuves existantes.

\section{Résumés des preuves sur la planification familiale}

- Accélérer l'adoption de la planification familiale volontaire et fondée sur les droits dans les pays en développement (vue d'ensemble) (mise à jour octobre 2018)

- Financement de la planification familiale (mise à jour octobre 2018)

- Réduction des grossesses précoces et involontaires chez les adolescentes (mise à jour octobre 2018)

- Amélioration de la prestation de services de planification familiale pendant les crises humanitaires

- Assurer la sécurité des contraceptifs par des chaînes d'approvisionnement efficaces

- Élargissement du choix des contraceptifs (mise à jour octobre 2018)

- Partenariat avec le secteur privé pour renforcer la fourniture de la contraception

Seuls les auteurs sont responsables des opinions exprimées dans cet article qui ne représentent pas nécessairement les opinions, décisions ou politiques des institutions avec lesquelles ils sont affiliés.

La présente traduction n'a pas été établie par l'Organisation mondiale de la Santé (OMS). L'OMS ne saurait être tenue pour responsable du contenu ou de l'exactitude de la présente traduction. L'édition originale anglaise est l'édition authentique qui fait foi.

Family Planning Evidence Brief-Accelerating uptake of family planning: WHO/RHR/17.07

Family Planning Evidence Brief-Partnering with the private sector: WHO/RHR/17.08

Family Planning Evidence Brief-Ensuring contraceptive security through effective supply chains: WHO/RHR/17.09

Family Planning Evidence Brief-Reducing early and unintended pregnancies among adolescents: WHO/RHR/17.10

Family Planning Evidence Brief-Reducing early and unintended pregnancies among adolescents: programme priorities: WHO/ RHR/17.11

Family Planning Evidence Brief-Ensuring adequate financing of family planning commodities and services: WHO/RHR/17.12

Family Planning Evidence Brief-Improving family planning service delivery in humanitarian crises: WHO/RHR/17.13

Family Planning Evidence Brief-Expanding contraceptive choice: WHO/RHR/17.14

Ce document a été financé par UK AID du gouvernement britannique toutefois, les opinions exprimées ne reflètent pas nécessairement les politiques du gouvernement britannique.

Préparé juillet 2017 